\title{
Development and Control of a Three DOF Planar Induction Motor
}

\author{
Masaaki Kumagai and Ralph L. Hollis
}

\begin{abstract}
This paper reports a planar induction motor that can output $70 \mathrm{~N}$ translational thrust and $9 \mathrm{Nm}$ torque within response time of $10 \mathrm{~ms}$. This motor consists of three linear induction armatures with vector control driver and three optical mouse sensors. First, an idea to combine linear induction motor is proposed. The power distribution to each element is derived from position and orientation of it. Then, developed system and its measured characteristics follow. Experimental results indicate potential of its direct drive feature.
\end{abstract}

\section{INTRODUCTION}

Omnidirectional actuation is one of desirable capabilities for mobile robots. Most of those robots achieve omnidirectional motion by using special wheels connecting to general rotary motors through reduction gears. Though these configurations work well, two major limitations arise. One is transmission error such as backlash that degrades accuracy and delay in response. The other is slip due to limitation in friction force between the wheels and a floor where the robot is on, which limit maximum acceleration of the robot. To solve these two problems, direct-drive planar actuator is favorable.

Many of planar actuators were developed by applying principles of rotary motors. For example, Lauwers et al. reported a robot with planar actuator based on stepper motor[1], which archived very precise positioning. Several types of planar motors were surveyed in a report[2].

One group of planar motors in based on induction motor. A linear induction motor (LIM) is one of successful linear motor that is a combination of an armature and a reaction plate, which is rather simple motor. By using more than two motors, at least, 3-DOF (translation and rotation) planar motor can be achieved. Dittrich et al. developed planar induction motor on such idea[3]. They used four LIMs for their motor, and measured steady characteristics. They also formed a closed loop for position control. An interesting motor was proposed by Fujii et al.[4]. It had a circular shape armature, and windings at a part of the arc work as an individual curved linear motor. They reported advantage in efficiency than simple combination of LIMs, whereas there is no freedom in armature arrangement. However, these works did not mention dynamic control or response while these features are important for robot control.

Our motivation is to develop and control the planar induction motor (PIM) with sufficient dynamic response. We already reported planar motion detection method using

Masaaki Kumagai is with the Faculty of Engineering, Tohoku Gakuin University, Tagajo 985-8537 Japan. Ralph L. Hollis is with The Robotics Institute, Carnegie Mellon University, Pittsburgh, PA 15213, USA

kumagai@tjcc.tohoku-gakuin.ac.jp,

rhollis@cs.cmu.edu

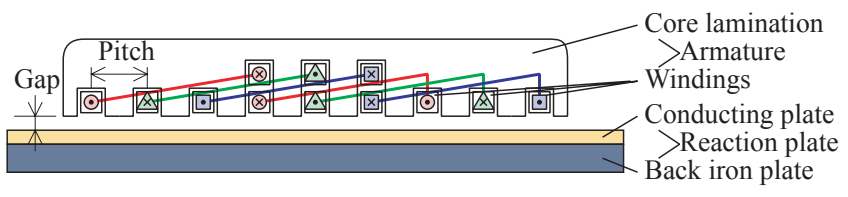

Fig. 1. A model of linear induction motor.

optical mouse sensors[5] and high-response LIM with own vector controller[6]. A PIM of up to $70 \mathrm{~N}$ translational thrust and $9 \mathrm{Nm}$ torque with $10 \mathrm{~ms}$ response was developed using these basis and control equations proposed in this paper. Experimental results include dynamic response of the system and position tracking ability even in $60^{\circ}$ inclined surface.

\section{METHODS OF PLANAR INDUCTION MOTOR}

We formed the planar induction motor (PIM) system with three linear induction motors (LIMs) for actuation and three laser mouse sensors for sensing. The control of each LIM is described first, and sensing method and overall control of the PIM follows.

\section{A. Control of the LIM}

A LIM is a kind of induction motor whose stator and rotor have straight shape as in Fig. 1, whereas a general (rotary) induction motor has cylindrical shape. One side of the motor is an armature that consists of a laminated core and a set of windings. The windings are usually grouped into three sets, forming three-phase coils. By supplying three-phase current to the coils, a traveling magnetic field is generated on the armature. The other side of the motor is a reaction plate that consists of a conducting plate and a back iron plate. The conducting plate is usually made of copper.

The model of the LIM is described by several equations though the characteristics of the LIM (electrical, magnetic, and dynamical) depends on the shape of lamination, windings, thickness of both the plates, and gap between armature and reaction plate. Because the detailed explanation of the model and equations requires pages[6], we show them briefly below:

$$
\begin{aligned}
f & \propto i_{0}(t) i_{q}(t) \\
i_{d}(t) & =i_{0}(t)+\frac{L}{R} \frac{d}{d t} i_{0}(t) \\
I_{0}(s) & =\frac{1}{1+(L / R) s} I_{d}(s) \\
i_{q}(t) & =\frac{L}{R} i_{0}(t) \omega_{c} \\
i & =\sqrt{i_{d}^{2}+i_{q}^{2}}
\end{aligned}
$$




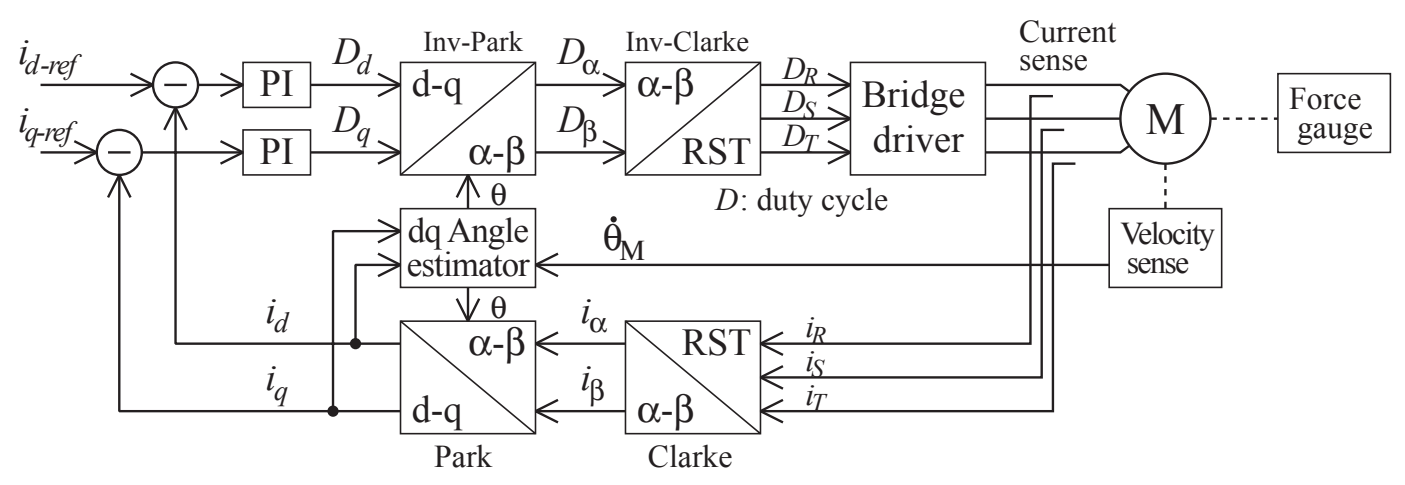

Fig. 2. Block diagram of vector control driver.

where $f$ is thrust force output of the LIM, $i_{0}(t)\left(I_{0}(s)\right.$ after Laplace transform) is magnetizing current, $i_{d}$ and $i_{q}$ are amplitude of two orthogonal components (cannot be measured but estimated) of supplied three-phase current whose amplitude and angular frequency are $i$ and $\omega_{c}$. The constant $L$ and $R$ is inductance and resistance parameter of the reaction plate that cannot be measured directly. The current term $i_{0}$ becomes constant in steady state if $i_{d}$ is kept constant because they have first-order lag relation. In that case $\left(i_{0}=i_{d}\right)$, the thrust of the LIM can be controlled by $i_{q}$.

Therefore, the control theory of the induction motor (not only the LIM but also rotary type) focuses on how to estimate and control $i_{d}$ and $i_{q}$, that is known as vector control. One hint for estimating them is (3), which is a relation between $i_{q}$ and $i_{0}$. It means that we can estimate $i_{d}\left(i_{0}\right)$ and $i_{q}$ if we know the ratio $L / R$.

By substituting (3) into (1), we obtain:

$$
F \propto i_{d} i_{q} \propto i_{0} i_{0} \omega_{c} .
$$

As mentioned above, the $i_{0}$ is usually kept constant by keeping $i_{d}$ constant in vector control method. In that case, the output force is proportional to $i_{q}$. It is also proportional to the frequency $\omega_{c}$ of the current, and square of the current.

The parameters $L$ and $R$ cannot be measured directly but we can estimate by a simple experiment. From two equations (1) and (4), we will obtain maximum output force when $i_{d}=i_{q}$ if we apply current of constant amplitude $i$. Hence we can estimate $L / R$ by finding where the force becomes maximum with $i_{d}=i_{q}$.

Figure 2 shows a block diagram of vector control LIM driver that we developed (Fig. 6). The actual current of the three-phase coils are measured, which are converted into $i_{d}$ and $i_{q}$ via rotating current vector $\left(i_{\alpha}, i_{\beta}\right)$. They are used for calculating $\omega_{c}$ and for estimation of rotation angle $\theta$ of the current. Then $i_{d}$ and $i_{q}$ are compared with reference command of those, and PI controller define the duty cycle applied for three-phase bridge driver via two inverse transformation. Note that above discussion about $\omega_{c}$ is in static state, and feed forwarded angular frequency $\theta_{M}$ is required based on relative speed between the armature and the reaction plate.
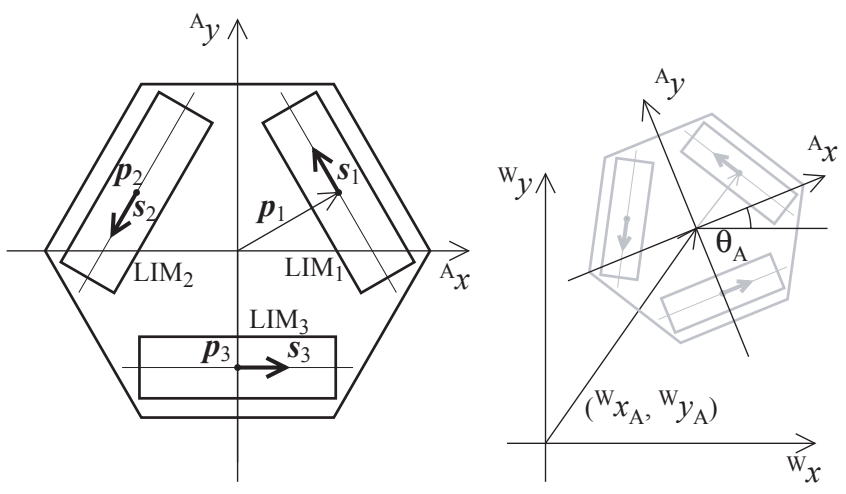

Fig. 3. A model of planar induction motor.

Concluding the description about the LIM, using an appropriate vector control, we need to decide $i_{d}, i_{q}$ to command the thrust output with supplement of relative speed between the armature and the reaction palate.

\section{B. 3-DOF Sensing of the PIM}

Two-dimensional motion of the PIM, i.e. translation in two orthogonal directions and rotation, is required for controlling motion of the PIM and deciding feed forward speed term of each LIM. The authors already proposed and confirmed motion sensing method using laser mouse sensors[5]. The method employs more than one optical (laser) mouse sensor that can sense surfaces velocity in two orthogonal directions. Combinations of each of three sensing values out of all sensors are translated into 3-DOF velocities. Then weighted mean of each results is calculated, which is integrated into position and rotational angle of the system.

Thus, this sensing system can provide position, rotational angle (orientation), translational velocity, and angular velocity of the PIM. The only problem is that it can detect relative motion but absolute position. The position and orientation drift as time goes by, but no large issue effects on the motion control. Detailed algorithms is described in previous report[5] (Note that the paper focused on sensing of ball rotation, but also mentioning planar motion sensing). 


\section{3-DOF Control of the PIM}

1) Calculation of force output: The PIM consist of three LIMs with motion sensors. Figure 3 shows arrangeement of LIMs. There is no need to arrange them symmetrically, but it is natural to do so. Let the position vector of each LIM (number $i=1,2,3$ ) be $\boldsymbol{p}_{i}=\left(p_{x i}, p_{y i}\right)$ and its thrust force generating direction vector $\boldsymbol{s}_{i}=\left(s_{x i}, s_{y i}\right), \quad\left|\boldsymbol{s}_{i}\right|=1$. We have two coordinate frames; world coordinate $W$ fixed on the reaction plate and local coordinate $A$ fixed on the PIM armature assembly. Let the origin of the frame $A$ (center of the PIM) be $\left({ }^{W} x_{A},{ }^{W} y_{A}\right)$ and its rotational angle be $\theta_{A}$. These $\left({ }^{W} x_{A},{ }^{W} y_{A}\right), \theta_{A}$ and their velocities are measured by above mouse sensors, and the mechanical design of the armature defines ${ }^{A} \boldsymbol{p}_{i}$ and ${ }^{A} \boldsymbol{s}_{i}$

The position and orientation of each LIM on coordinate $W$ is calculated first:

$$
\begin{aligned}
\left(\begin{array}{c}
{ }^{W} p_{x i} \\
{ }^{W} p_{y i}
\end{array}\right)= & \left(\begin{array}{rr}
\cos \theta_{A} & -\sin \theta_{A} \\
\sin \theta_{A} & \cos \theta_{A}
\end{array}\right)\left(\begin{array}{c}
{ }^{A} p_{x i} \\
{ }^{A} p_{y i}
\end{array}\right) \\
& +\left(\begin{array}{c}
{ }^{W} x_{A} \\
{ }^{W} y_{A}
\end{array}\right) \\
\left(\begin{array}{c}
{ }^{W} s_{x i} \\
{ }^{W} s_{y i}
\end{array}\right)= & \left(\begin{array}{rr}
\cos \theta_{A} & -\sin \theta_{A} \\
\sin \theta_{A} & \cos \theta_{A}
\end{array}\right)\left(\begin{array}{c}
{ }^{A} s_{x i} \\
{ }^{A} s_{y i}
\end{array}\right)
\end{aligned}
$$

Then the velocity at each LIM on $\mathrm{W}\left({ }^{W} v_{x i},{ }^{W} v_{y i}\right)$ is:

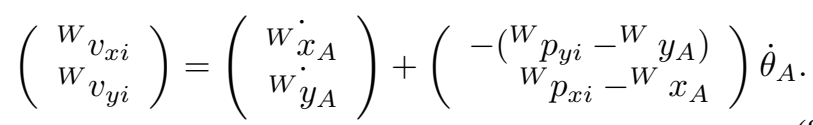

The magnitude of LIM velocity along its thrust axis $s_{i}$ is obtained by an inner product as follows.

$$
v_{s i}=\left({ }^{W} v_{x i},{ }^{W} v_{y i}\right) \cdot\left({ }^{W} s_{x i},{ }^{W} s_{y i}\right)
$$

This velocity component $v_{s i}$ is used for velocity feed forward in vector control of each LIM.

Next, a thrust output command for each LIM $f_{i}$ is calculated from force/torque command on world coordinate ${ }^{W} f_{x},{ }^{W} f_{y}, T$. All of following derivations are done on world coordinate, and we omit notation " $W$ " for simplicity.

The relation between the force outputs $f_{i}$ and total output of the PIM $f_{x}, f_{y}, T$ is approximated as follows.

$$
\begin{aligned}
f_{x} & =\sum_{i=1}^{3} f_{i} s_{x i} \\
f_{y} & =\sum_{i=1}^{3} f_{i} s_{y i} \\
T & =\sum_{i=1}^{3}\left\{\left(p_{x i}-x_{A}\right)\left(f_{i} s_{y i}\right)-\left(p_{y i}-y_{A}\right)\left(f_{i} s_{x i}\right)\right\} \\
& =\sum_{i=1}^{3}\left\{\left(p_{x i}-x_{A}\right) s_{y i}-\left(p_{y i}-y_{A}\right) s_{x i}\right\} f_{i} .
\end{aligned}
$$

The equation (12) comes from definition of torque $r \times$ $\left.\boldsymbol{f}\right|_{z}$ where $\boldsymbol{r}=\left(p_{x i}-x_{A}, \quad p_{y i}-y_{A}, 0\right)$ and $\boldsymbol{f}=$ $\left(f_{i} s_{x i}, f_{i} s_{y i}, 0\right)$. These equations can be expressed by matrix form:

$$
\begin{aligned}
\left(\begin{array}{c}
f_{x} \\
f_{y} \\
T
\end{array}\right)= & \left(\begin{array}{rrr}
s_{x 1} & s_{x 2} & s_{x 3} \\
s_{y 1} & s_{y 2} & s_{y 3} \\
t_{1} & t_{2} & t_{3}
\end{array}\right)\left(\begin{array}{l}
f_{1} \\
f_{2} \\
f_{3}
\end{array}\right) \\
= & C\left(\begin{array}{c}
f_{1} \\
f_{2} \\
f_{3}
\end{array}\right) \\
& t_{i}=\left(p_{x i}-x_{A}\right) s_{y i}-\left(p_{y i}-y_{A}\right) s_{x i}
\end{aligned}
$$

If the conversion matrix $C$ has the inverse, this equation can be solved to obtain force commands for LIMs $\left(f_{1}, f_{2}, f_{3}\right)$ from $\left(f_{x}, f_{y}, T\right)$.

$$
\left(\begin{array}{l}
f_{1} \\
f_{2} \\
f_{3}
\end{array}\right)=\boldsymbol{C}^{-1}\left(\begin{array}{c}
f_{x} \\
f_{y} \\
T
\end{array}\right)
$$

One aspect requiring confirmation is adequacy of approximating the LIM to be a single force output at the center point of the armature. The experimental results indicated that this approximation had no problem by measuring total output force/torque, while, needless to say, the actual output is distributed on the armature area facing to reaction plate.

Another concern about above calculation is extension to the case that we have more than three LIMs. One solution is using techniques such as pseudo inverse matrix. One might want to minimize $\sum\left|f_{i}\right|$ or $\sum\left|f_{i} v_{s i}\right|$, which reduces power consumption. However we need to consider the force ripple of the LIM. Different from a rotary induction motor, a LIM has "end effect" due to discontinuity at both side. The output force of the LIM fluctuates even it is supplied AC current with constant frequency and constant amplitude. This force ripple synchronizing to current frequency adversely affects the control of the system, which is noticeable especially in lower frequency. To avoid this effect, one solution is to use only three major LIMs and others help them in case much more output is required.

2) Position control of the PIM: On the world coordinate, we applied simple PID control for position and PD control for rotation.

$$
\begin{aligned}
e_{x(y)} & =x(y)_{A, r e f}-x(y)_{A, a c t} \\
f_{x(y), c m d} & =K_{P P} e_{x(y)}+K_{P D} \frac{d e_{x(y)}}{d t}+K_{P I} \int e_{x(y)} d t \\
e_{\theta} & =\theta_{A, r e f}-\theta_{A, a c t} \\
T_{c m d} & =K_{T P} e_{\theta}+K_{T D} \frac{d e_{\theta}}{d t},
\end{aligned}
$$

where $K \mathrm{~s}$ are constant gain (turned empirically in experiments) and ref, act, and cmd denote reference and measured position and command for the PIM. We used PID for position so that it can respond to bias such as gravity.

\section{IMPLEMENTATION AND EXPERIMENTS}

We materialized a PIM with maximum translational force of $70 \mathrm{~N}$ and torque of $9 \mathrm{Nm}$. The PIM was successfully controlled in closed loop with mouse position sensing. The hardware and experimental results are described. 


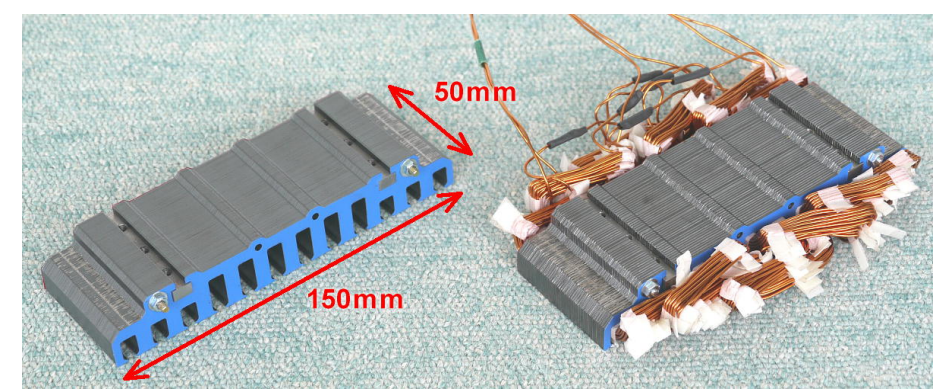

(a) LIM armature

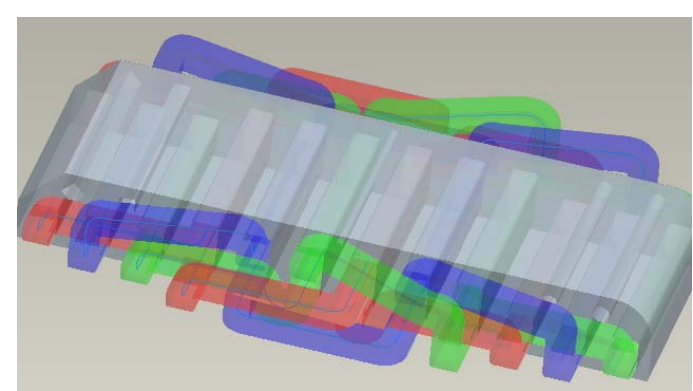

(b) Three phase model of windings

Fig. 4. Developed linear induction armature.

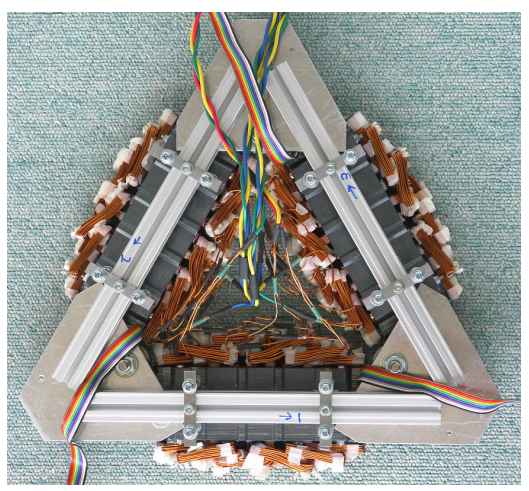

(a) Top view

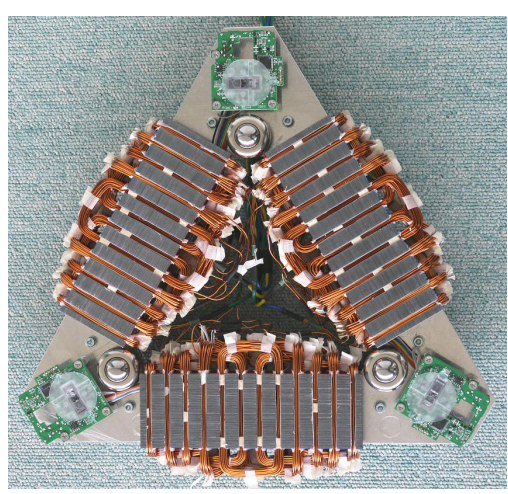

(b) Bottom view

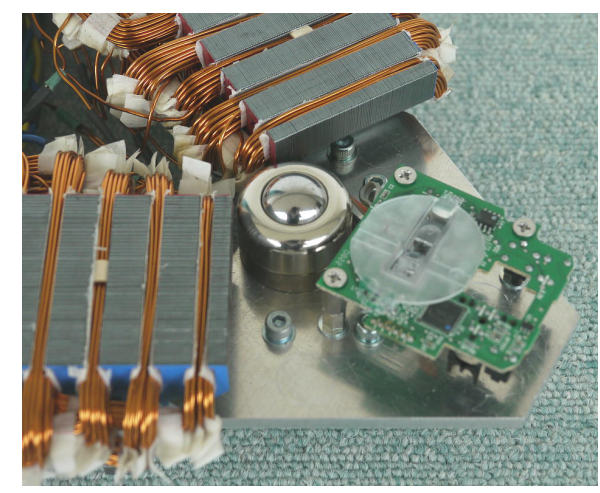

(c) Corner part

Fig. 5. Developed planar induction motor.

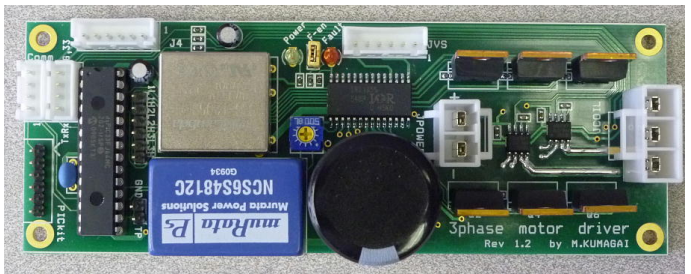

Fig. 6. Three-phase vector control driver for LIM armature.

\section{A. Implementation of the PIM}

The LIM used for the PIM was also our own design, which is shown first. Then the PIM follows.

1) LIM: We developed the three-phase LIM armature shown in Fig. 4 whose length, width, and height is $150 \mathrm{~mm}$, $50 \mathrm{~mm}$ and $23 \mathrm{~mm}$, and weighs $1.2 \mathrm{~kg}$. It can output $40 \mathrm{~N}$ in our PIM setting, but $70 \mathrm{~N}$ in extreme condition for short time (the limitation is temperature rise, stiffness of a supporting frame and the reaction plate, and gap). The armature has three poles with 12 slots. Fig. 4(b) shows the schematic model of the three groups of windings, which was designed so that the windings become compact to arrange as PIM and that three groups have similar characteristics to reduce ripples. Three summations of three groups consisting of three windings in series had almost equal inductance, whose difference is less than $2 \%$. The core material is magnetic steel sheet of $0.5 \mathrm{~mm}$ (non-oriented JIS 50A $470^{1}$ ) laminating 100 sheets, which weighs $0.75 \mathrm{~kg}$. Each winding is 25 turns coil of $1 \mathrm{~mm}$ diameter magnetic wire (total 75 turns in one phase). Three

${ }^{1}$ This code means 50 micron and core loss of $4.7 \mathrm{~W} / \mathrm{kg}$ at $1.5 \mathrm{~T} 50 \mathrm{~Hz}$. circuits of windings form $\Delta$ connection.

The LIM is controlled by our original vector controller in Fig. 6. The control cycle was $0.1 \mathrm{~ms}(10 \mathrm{kHz})$.

2) PIM: Figure 5 shows the PIM armature consisted of three LIM armatures, three ball transfers to support the frame, and three mouse sensors (Avago ADNS6010 obtained from Microsoft X5 mouse). All the components are arranged symmetrically. The LIM armature is positioned on a side of regular triangle and is $80 \mathrm{~mm}$ distant from the center. The gap between armature and the plate was $0.5 \mathrm{~mm}$. The ball transfers and sensors are located on a circle of $105 \mathrm{~mm}$ and $140 \mathrm{~mm}$ radius each. These mechanical parameters are used for PIM force/torque control and position/rotation measurement. Whole system is size of $180 \mathrm{~mm}$ in diameter and weighs $4.8 \mathrm{~kg}$. Three LIM vector controller and 50V-20A power source was located outside of the PIM while mouse sensor controller was on it. The LIM controllers and sensors were connected to a PC running Windows xp via serial port. The PIM control period was set to $10 \mathrm{~ms}(100 \mathrm{~Hz})$.

\section{B. Experiments}

1) Characteristics of LIM: We measured dynamic characteristic of the LIM first. Figure 7(a) shows the linearity of the force output. In this experiment, $i_{d}$ is set to be $11 \mathrm{~A}(10000$ in internal unit of the controller), and $i_{q}$ was increased by $1.1 \mathrm{~A} \mathrm{(1000)} \mathrm{step} \mathrm{at} 100 \mathrm{~ms}$ to $33 \mathrm{~A}$ (30000). With constant $i_{d}$, the output force is theoretically proportional to $i_{q}$, which is confirmed on this experiment. Note that the force increased faster a bit than proportional line extrapolating from lower output range in the former half. This is due to mechanical 


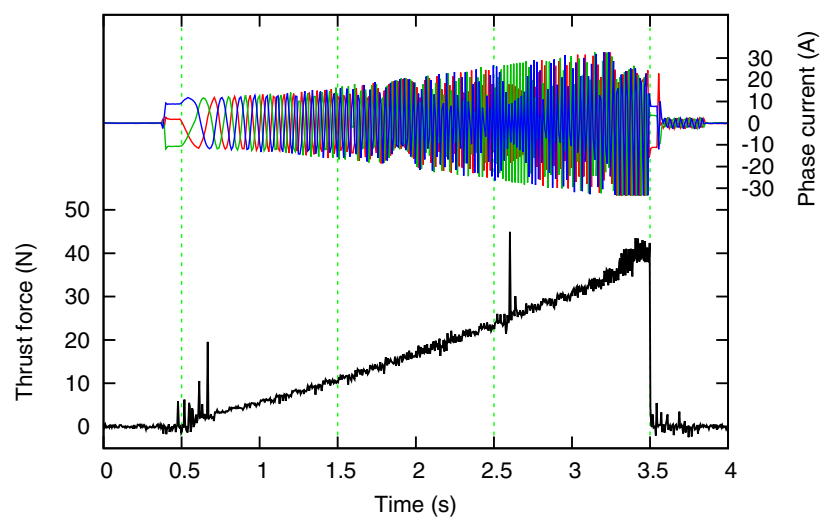

(a) linearly swept $i_{q}$ (0 to 33A) with constant $i_{d}(11 \mathrm{~A})$

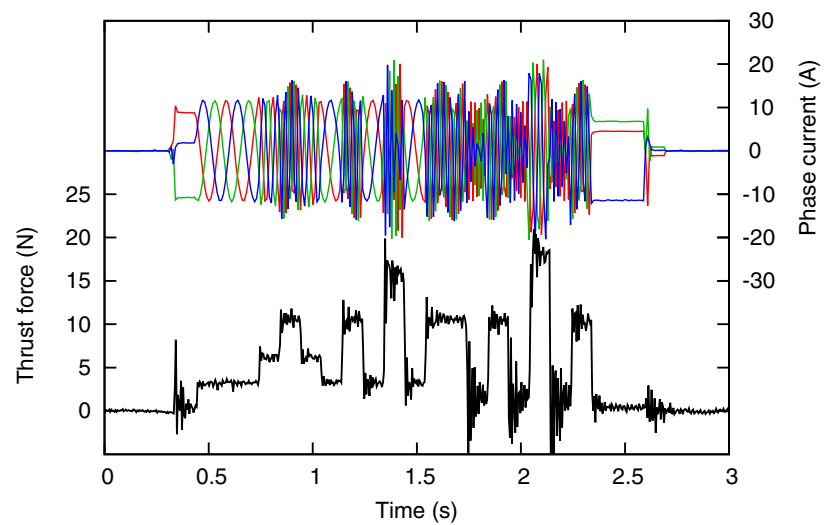

(b) step response of force output

Fig. 7. Force output of a LIM and three phase currents. Note that the currents were saturated at last part of sweep, and that the waveforms of the currents were affected by sampling theorem, which degraded the forms.

stiffness that caused decrease of the gap by increase of attraction force between armature and the back iron plate. Smaller gap generates larger force with the same current.

Figure 7(b) shows dynamic response of the LIM. Before $1.6 \mathrm{~s}$, the $i_{d}$ is kept constant at 10000 and $i_{q}$ is changed through 2000, 5000, 10000, and 150000 in the unit at $100 \mathrm{~ms}$ interval. In the latter part, $i_{q}$ is kept constant at 10000 and $i_{d}$ is changed. As mentioned before, $i_{d}$ is usually kept constant. In the former half, the force output respond in $10 \mathrm{~ms}$ with small ripple, whereas larger ripple in the latter half especially the case decreasing $i_{d}$. These results confirmed that we can achieve $10 \mathrm{~ms}$ response only by commanding $i_{q}$.

2) Position and Orientation Control: We confirmed the force and torque output of the PIM using (15). The translational force could be set independent from the orientation of the PIM. Then we carried out position and orientation tracking control including confirmation of position sensing, force/torque output control.

The reference was designed; the target of center of the PIM was square shaped without interpolation, i.e. the reference jumped to the next point periodically to confirm step response in $\mathrm{x}$ and $\mathrm{y}$ direction; the reference of rotational angle is generated by sinusoidal function. Figure 8 shows the experimental results of the position tracking. These experiments are also included in accompanied video, which are clearer to check the motion and response. In Fig. 8(a), only the position was set to $\pm 20 \mathrm{~mm}$. In (b) the orientation of the PIM is changed in 0.4rad $\left(23^{\circ}\right)$ amplitude, which indicated the change of orientation did not affect a lot to position control. In (a) and (b) the period of the motion was $4 \mathrm{~s}$, whereas $1 \mathrm{~s}$ in (c). The rotational amplitude is the same while position amplitude was decreased to $10 \mathrm{~mm}$. It was still under control but the error increased in (c). In the last case (d), the reaction plate with the PIM was inclined by hand up to over $60^{\circ}$. Because the position control employed I control, the gravity term was almost compensated. Note that a wheeled robot slips down from such a steep slope but the PIM can generate thrust force not using friction, and also stick to the surface. In the case (a) to (c), the averaged power consumption was approximately $250 \mathrm{~W}$, and near $1 \mathrm{~kW}$ while most tilted condition in (d). These results showed usefulness of our PIM and the method.

\section{CONCLUSIONS}

A planar induction motor was developed, and control methods using vector control was described in this paper. The hardware consisted of three linear induction motor armatures for actuation, three optical mouse sensors for position and velocity sensing, and three ball transfers for support. The vector controller of each LIM had response speed faster than $10 \mathrm{~ms}$, which enabled good position tracking ability. We achieved closed loop position tracking and measured dynamic response in experiments. At most extreme cases, the speed of the PIM reached $450 \mathrm{~mm} / \mathrm{s}$ in less than $0.1 \mathrm{~s}$ with peak acceleration of $12 \mathrm{~m} / \mathrm{s}^{2}$. The PIM armature output approximately $60 \mathrm{~N}$ at that time. Also, the tracking experiment was carried out on steep slope of $60^{\circ}$ inclination, which resulted in almost same capability. The developed PIM will be one of probable omnidirectional actuator even though it requires reaction plate for operating area. We focus on application of the PIM in the next work.

\section{ACKNOWLEDGEMENTS}

A part of this work was performed in the Microdynamic Systems Laboratory, The Robotics Institute, Carnegie Mellon University, as a part of the dynamically stable mobile robots project. It was also supported by KAKENHI(11022515).

\section{REFERENCES}

[1] T.B. Lauwers, Z.K. Edmondson, R.L. Hollis, "Free-Roaming Planar Motors: Toward Autonomous Precision Planar Mobile Robots", ICRA 2004, pp. 4498-4503

[2] J. Pan, N.C. Cheung, J. Yang, "Structure and characteristics of closedloop two-dimensional surface motors - a literature survey", proc. Power Electronics and Drive Systems 2003, 2003, pp.236-241

[3] P. Dittrich, D. Radeck, "3-DOF Planar Induction Motor", proc. Electro/Information Technology 2006, 2006, pp.81-86

[4] N. Fujii, M. Fujitake, "Two-dimensional drive characteristics by circular-shaped motor", IEEE trans. Industrial Application, vol.35, no.4, 2002, pp.803-809

[5] M. Kumagai, R.L. Hollis, "Development of a three-dimensional ball rotation sensing system using optical mouse sensors", ICRA 2011, pp. 5038-5043

[6] M. Kumagai: "Development of a Linear Induction Motor and a Vector Control Driver", SICE tohoku chapter workshop material, 2010, pp. 262-9 (in Japanese language) 

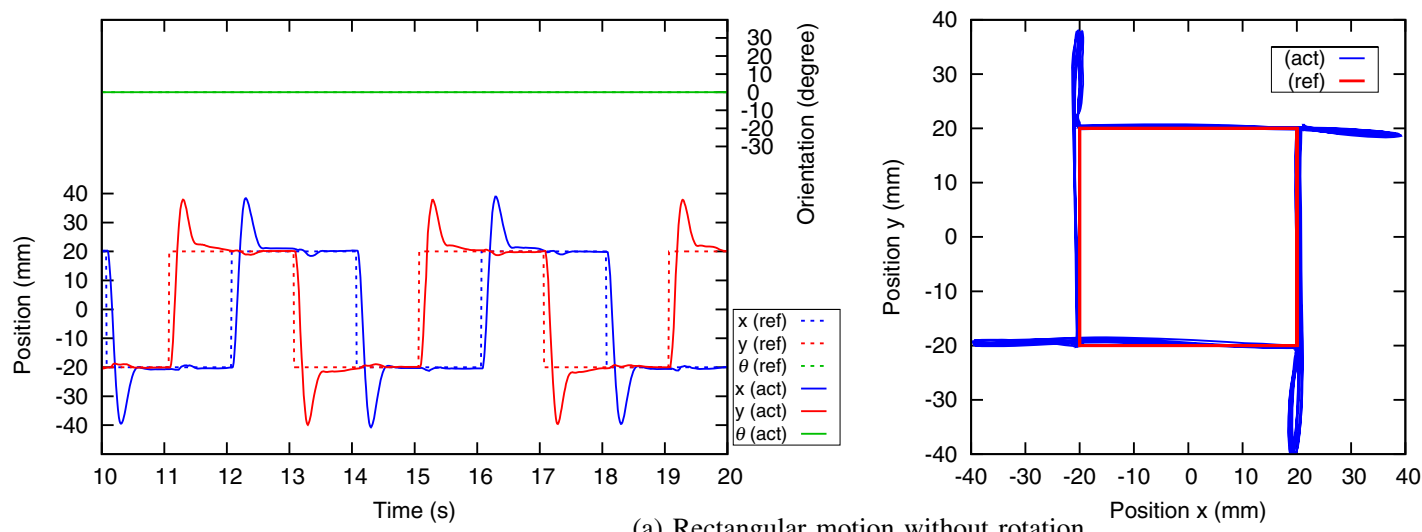

Conditions

position: $\pm 20 \mathrm{~mm}$

orientation: 0

period: 4

Notes

max speed: $450 \mathrm{~mm} / \mathrm{s}$ max accel.: $12 \mathrm{~m} / \mathrm{s}^{2}$
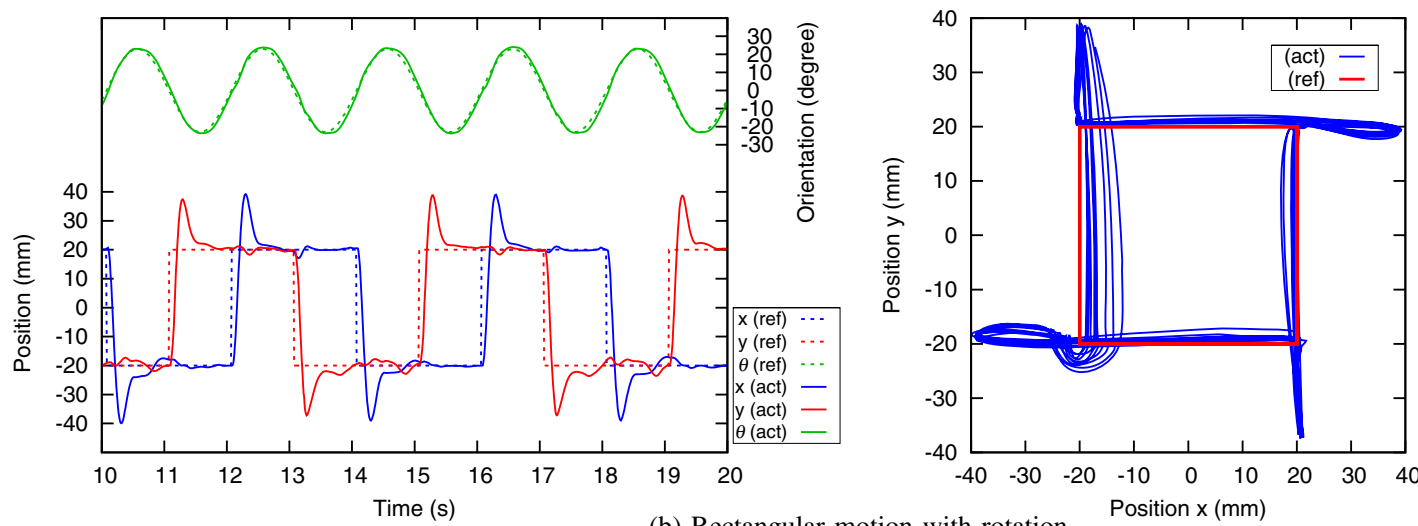

Conditions:

position: $\pm 20 \mathrm{~mm}$ orientation: $\pm 0.4 \mathrm{rad}$ $\left( \pm 23^{\circ}\right)$

period: $4 \mathrm{~s}$

Notes

max speed: $450 \mathrm{~mm} / \mathrm{s}$ $\max$ accel.: $10 \mathrm{~m} / \mathrm{s}^{2}$
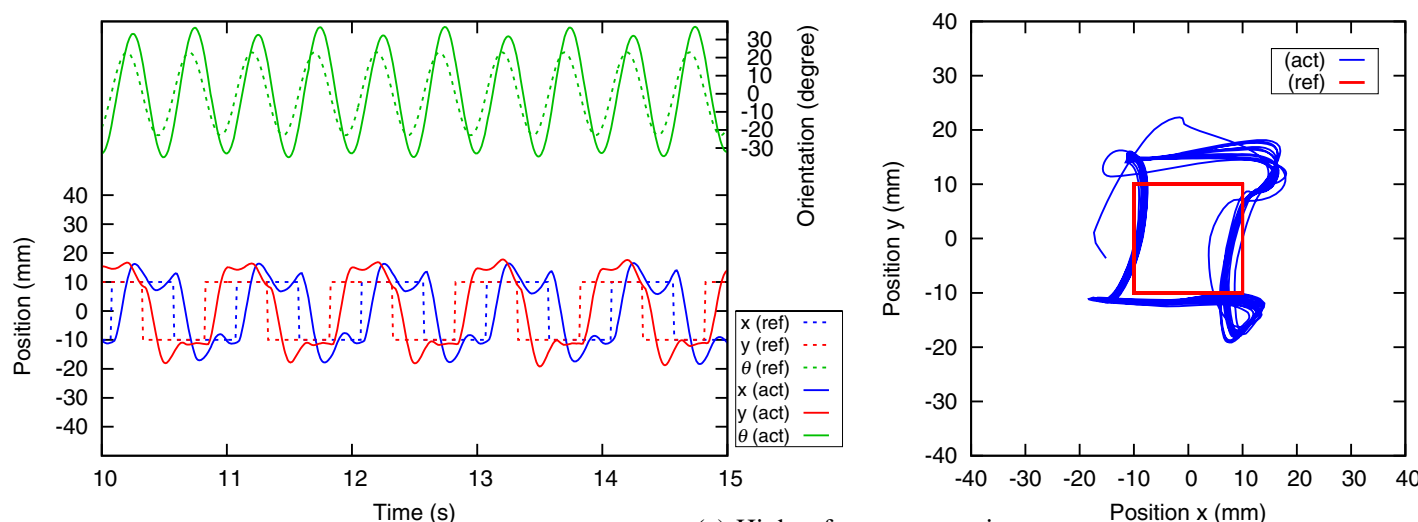

Conditions:

position: $\pm 10 \mathrm{~mm}$ orientation: $\pm 0.4 \mathrm{rad}$ $\left( \pm 23^{\circ}\right)$ period: $\quad 1 \mathrm{~s}$

Notes

max speed: $250 \mathrm{~mm} / \mathrm{s}$ $\max$ accel.: $8 \mathrm{~m} / \mathrm{s}^{2}$

(c) Higher frequency motion
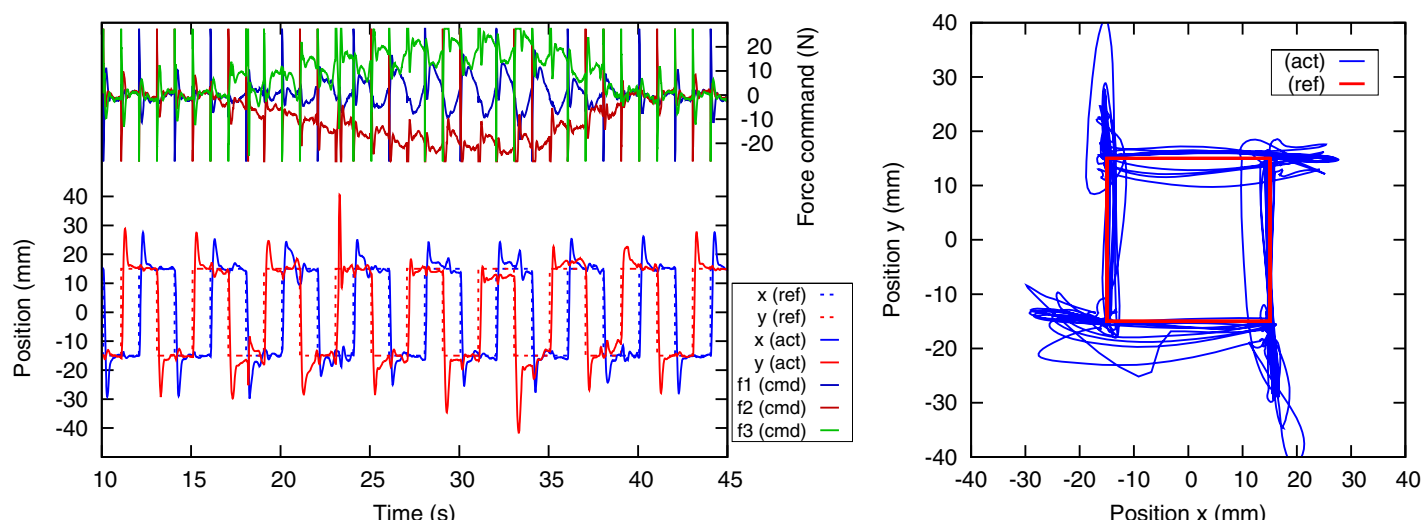

Conditions:

position: $\pm 15 \mathrm{~mm}$ orientation: $\pm 0.4 \mathrm{rad}$ $\begin{array}{ll} & \left( \pm 23^{\circ}\right) \\ \text { period: } & 4 \mathrm{~s}\end{array}$

Notes

$\max$ tilt: $>60^{\circ}$

(d) Rectangular motion with rotation while tilting the plate about $x$ axis (gravity acts on $y$ direction)

Fig. 8. Time responses and geometrical plots of PIM in four conditions. '( ref $)$ ', '( act)' and '( $c m d)$ ' mean reference value, measured actual value, and commanded value. Maximum speed and acceleration is typical value measured from the data. 